\title{
Ubiquitin ligases HUWE1 and NEDD4 cooperatively control signal-dependent PRC2-Ezh1 $1 / \beta$-mediated adaptive stress response pathway in skeletal muscle cells
}

\author{
Peng Liu', Muhammad Shuaib ${ }^{1}$, Huoming Zhang ${ }^{2}$, Seba Nadeef ${ }^{1}$ and Valerio Orlando ${ }^{1 *}(\mathbb{0}$
}

\begin{abstract}
Background: While the role of Polycomb group protein-mediated "cell memory" is well established in developmental contexts, little is known about their role in adult tissues and in particular in post-mitotic cells. Emerging evidence assigns a pivotal role in cell plasticity and adaptation. PRC2-Ezh1a/ $\beta$ signaling pathway from cytoplasm to chromatin protects skeletal muscle cells from oxidative stress. However, detailed mechanisms controlling degradation of cytoplasmic Ezh1 $\beta$ and assembly of canonical PRC2-Ezh1 a repressive complex remain to be clarified.

Results: Here, we report NEDD4 ubiquitin E3 ligase, as key regulator of Ezh1 $\beta$. In addition, we report that ubiquitination and degradation of Ezh $1 \beta$ is controlled by another layer of regulation, that is, one specific phosphorylation of serine 560 located at Ezh1 $\beta$-specific C terminal. Finally, we demonstrate that also Ezh1 a needs to be stabilized under stress condition and this stabilization process requires decreased association pattern between another E3 ubiquitin ligase HUWE1.
\end{abstract}

Conclusions: Together, these results shed light on key components that regulate PRC2-Ezh $1 \mathrm{a} / \beta$ pathway to direct modulation of epigenome plasticity and transcriptional output in skeletal muscle cells.

Keywords: HUWE1, NEDD4, Polycomb, Ubiquitination

\section{Background}

Besides its role in developmental memory, in adult postmitotic cells epigenome structure does not appear to be a rigid platform, but rather a dynamic system allowing plasticity to adapt transcriptional programs to naturally changing environmental cues.

Polycomb group proteins (PcG) and the role of H3K27me3 modification in maintaining cellular memory is well known [1-4]. Most of these studies focused on the

\footnotetext{
*Correspondence: Valerio.orlando@kaust.edu.sa

${ }^{1}$ BESE Division, KAUST Environmental Epigenetics Program,

King Abdullah University Science and Technology (KAUST),

Thuwal 23955-6900, Saudi Arabia

Full list of author information is available at the end of the article
}

contribution of Ezh2 histone methyltransferase (HMT) mediating H3K27 methylation. However, mammalian cells contain a second potential H3K27 HMT, Ezh1, highly related to Ezh2. Interestingly, Ezh1 is expressed mostly in embryonic stem cells, in combination with Ezh2, and in adult post-mitotic tissues where Ezh2 is absent [5-7]. In pluripotent cells, Ezh1 could compensate for Ezh2 role in mediating H3K27 methylation both in vitro and in vivo $[5,6,8,9]$. However, biochemical studies indicate Ezh1 as a weak HMT and its role in post-mitotic cells appears to be complex. Indeed, genome wide studies in skeletal muscle cells provided clues about direct association of Ezh1 with active promoters overlapping with H3K4me3-enriched regions [7, 10, 11] (not H3K27m3) and required for RNA Pol II elongation [10].

(c) The Author(s) 2019. This article is licensed under a Creative Commons Attribution 4.0 International License, which permits use, sharing, adaptation, distribution and reproduction in any medium or format, as long as you give appropriate credit to the original author(s) and the source, provide a link to the Creative Commons licence, and indicate if changes were made. The images or other third party material in this article are included in the article's Creative Commons licence, unless indicated otherwise in a credit line to the material. If material is not included in the article's Creative Commons licence and your intended use is not permitted by statutory regulation or exceeds the permitted use, you will need to obtain permission directly from the copyright holder. To view a copy of this licence, visit http://creativeco mmons.org/licenses/by/4.0/. The Creative Commons Public Domain Dedication waiver (http://creativecommons.org/publicdomain/ zero/1.0/) applies to the data made available in this article, unless otherwise stated in a credit line to the data. 
However, we previously reported a novel molecular mechanism in skeletal muscle tissue physiology showing the role of PRC2-Ezh1 in modulating H3K27me3 epigenome plasticity in response to oxidative stress [12]. In detail, we found that Ezh1 produced two different isoforms, Ezh $1 \alpha$ and Ezh1 $\beta$ exhibiting nucleus and cytosol exclusive localization, respectively. Under oxidative stress or atrophic conditions, Ezh1 $\beta$ is degraded through $26 \mathrm{~S}$ proteasome system. In this way, EED will escape sequestration by Ezh1 $\beta$ and shuttle from cytosol to nucleus to give rise to canonical PRC2-Ezh1 repressive complex through the interaction with Ezh1 $\alpha$ and SUZ12. This triggers Ezh1-dependent H3K27me3 signature and gene silencing at whole genome scale, allowing post-mitotic cells to adapt to oxidative stress thus, unveiling the plastic nature of PRC2-Ezh1 regulation. These findings suggest that Ezh1 is involved in different aspects of transcriptional regulation, both activation and repression, through canonical PRC2-Ezh1 or non-canonical PRC2-Ezh1 pathways. This duality suggests a complex regulated activity of Ezh1 function.

Stoichiometric regulation of PRC2 components is an essential feature of PcG physiology [2]. In different contexts, the role of post-translation modifications (PTMs) in regulating Ezh2 stability, intracellular dynamics and activity has been reported. Smurf2-mediated K421 ubiquitination of Ezh2 and degradation of Ezh2 facilitates hMSC neuron differentiation [13]. Recent data described Praja1 Ubiquitin ligase regulates stability of Ezh2 in a p38 signaling-dependent manner during skeletal myogenesis $[14,15]$. Many different ubiquitin E3 ligases have been reported to control Ezh2 in different tumor types [1618]. These include Trcp1/FBXW1, FBXW7 and TRAF6 $[16,18,19]$. Ubiquitination and degradation of Ezh2 were modulated in a phosphorylation-dependent manner $[15,18,19]$. These findings clearly highlight how different PTMs work cooperatively to regulate activity of Ezh2 under normal differentiation process or tumor cell types.

The mechanisms and role of PTMs in PRC2-Ezh1 dynamics in adult tissues allowing epigenetic adaptive stress response are not known. Here, we report about the ubiquitin ligase dependent signaling dynamics of PRC2-Ezh $\alpha / \beta$ pathway in skeletal muscle cells. We show that NEDD4 is the major ubiquitin E3 ligase to mediate Ezh1 $\beta$ ubiquitination and degradation. Further, we identify Serine 560 phosphorylation as the signal essential for Ezh1 $\beta$ Ub-E3-ligase dependent degradation. More surprisingly, Ezh1 $\alpha$ also exhibited a degradation dynamics in response to stress. We show that Ezh $1 \alpha$ requires stabilization under oxidative stress condition to facilitate canonical PRC2-Ezh1 $\alpha$ efficient assembly and this function is controlled by HUWE1 ubiquitin E3 ligase. Overall, our data identify NEDD4 and HUWE1, as the key players working in cooperation to regulate the stability of Ezh1 $\beta$ and Ezh $1 \alpha$, respectively, allowing PcG-dependent epigenetic adaptive response in skeletal muscles.

\section{Results \\ Identification of ubiquitin E3 ligases associated with Ezh1 $\beta$ under oxidative stress condition}

Our previous work demonstrated that, in response to oxidative stress, Ezh1 $\beta$ would undergo increased ubiquitination and degradation by $26 \mathrm{~S}$ proteasome pathway [12].

To identity candidate E3 ligases associated with Ezh1 $\beta$, we used tandem affinity purification (TAP) strategy [20]. We constructed $\mathrm{C} 2 \mathrm{C} 12$ cell line constitutively expressing Ezh1 $\beta$ tagged with FLAG and HA at its $C$ terminal. Sub-localization of fusion protein Ezh1 $\beta$-FLAG-HA was determined in myoblasts and differentiated myotubes. Immunostaining experiments clearly showed that Ezh1 $\beta$ FLAG-HA localized within cytosol, which is consistent with endogenous Ezh1 $\beta$ localization pattern as previously reported [12] (Additional file 1: Fig. S1a). We also checked relative expression level of Ezh1 $\beta$-FLAG-HA compared with the endogenous Ezh1 $\beta$ protein. Expression level of exogenous Ezh1 $\beta$-FLAG-HA was similar to endogenous Ezh1 $\beta$ level (Additional file 1: Fig. S1b). We noticed that in addition to one specific Ezh1 $\beta$-FH band as predicted molecular weight, another two high molecular bands were detected. To verify whether these two bands were containing Ezh1 $\beta$ polypeptide, we cut these two bands and sent them for mass spectrometry (MS) analysis. Ezh1 $\beta$ was highly represented with highest score in our MS analysis list (Additional file 2: Datasheet 1), confirming that these two bands are also Ezh1 $\beta$-FH specific, although the reason remains to be elucidated. Further, in vivo ubiquitination assay was performed using Ezh1 $\beta$-FLAG-HA stable cell line. The results clearly show poly-ubiquitination of Ezh1 $\beta$ levels increased upon oxidative stress condition and exhibit as typical smear profile (Additional file 1: Fig. S2).

Following $\mathrm{H}_{2} \mathrm{O}_{2}$ treatment, all HA elute samples were subjected to SDS-PAGE and silver staining (Fig. 1a). In comparison with $\mathrm{C} 2 \mathrm{C} 12$ wild-type mock samples, many specific protein partners were immunoprecipitated through TAP assay (Fig. 1a). Then, HA elutes from both Ezh1 $\beta$-FH stable cell line and wild-type $\mathrm{C} 2 \mathrm{C} 12$ cell line were sent for mass spectrometry (MS) analysis and immunoprecipitated interacting protein partners were listed (Additional file 3: Datasheet 2). Specificity was confirmed by EED resulting as top interacting partner of Ezh1 $\beta$ in three independent experiments (Additional file 3: Datasheet 2 and Fig. 1b). Ubiquitin E3 ligases, HUWE1, NEDD4 and CUL7/FBXW8 were identified through this TAP strategy (Additional file 3: Datasheet 2 and Fig. 1b). The specificity of these interactions was 


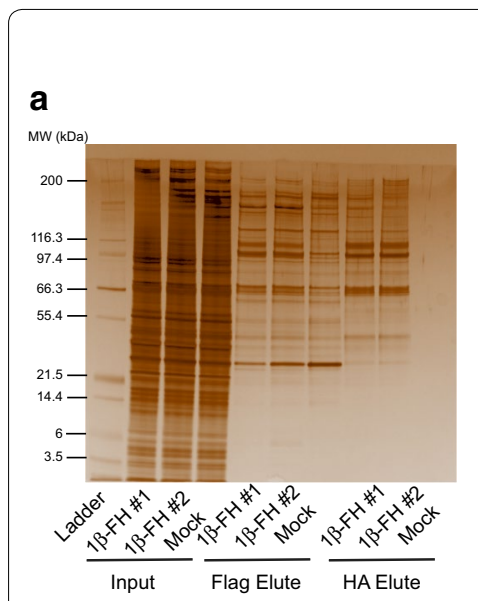

\begin{tabular}{|c|c|c|c|}
\hline b & Protein & Mascot score & $\begin{array}{l}\text { Number of } \\
\text { unique peptid }\end{array}$ \\
\hline \multirow{6}{*}{$\begin{array}{l}\stackrel{\Xi}{\mathbb{0}} \\
\frac{.0}{0} \\
\overline{0} \\
\square\end{array}$} & Ezh1 $\beta$ & 11948 & 56 \\
\hline & EED & 383 & 12 \\
\hline & CUL7 & 724 & 16 \\
\hline & HUWE1 & 344 & 11 \\
\hline & NEDD4 & 106 & 3 \\
\hline & FBXW8 & 117 & 2 \\
\hline \multirow{6}{*}{ 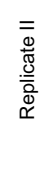 } & Ezh1 $\beta$ & 13530 & 67 \\
\hline & EED & 1024 & 38 \\
\hline & CUL7 & 434 & 12 \\
\hline & HUWE1 & 30 & 1 \\
\hline & NEDD4 & 69 & 3 \\
\hline & FBXW8 & 71 & 2 \\
\hline \multirow{6}{*}{ 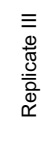 } & Ezh1 $\beta$ & 9614 & 53 \\
\hline & EED & 683 & 14 \\
\hline & CUL7 & 829 & 21 \\
\hline & HUWE1 & 336 & 13 \\
\hline & NEDD4 & 137 & 3 \\
\hline & FBXW8 & 88 & 3 \\
\hline
\end{tabular}

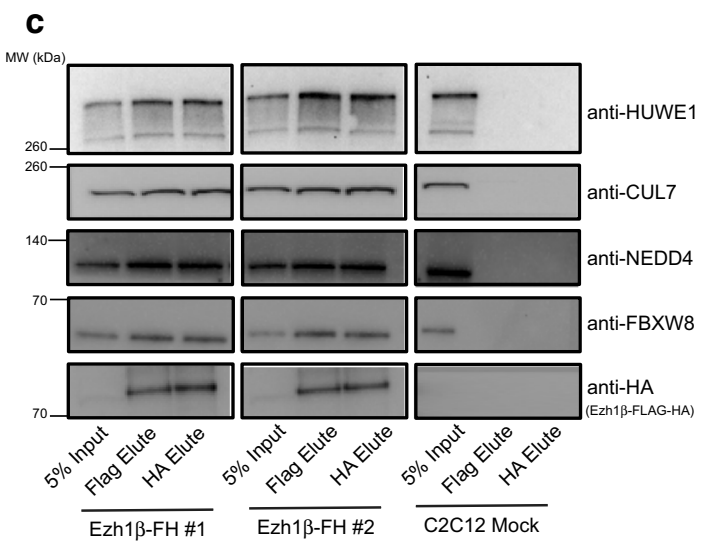

Fig. 1 Immunoprecipitation of Ezh1 $\beta$-associated protein partners under oxidative stress condition. a Ezh1 $\beta$-FH-associated protein complexes were tandemly affinity purified from cytosolic extracts of $\mathrm{C} 2 \mathrm{C} 12$ stable cell line which expresses C-terminally FLAG-HA tagged Ezh1 $\beta$. Flag and HA elutes indicate samples eluted with FLAG and HA peptides, respectively. Flag and HA elute samples were separated by SDS-PAGE and silver stained. Cytosolic protein extracts from C2C12 wild-type cell line were used as mock. Ezh1 $\beta$-FH expressing C2C12 and normal C2C12 myotube samples, after changing differentiation medium for 2 days, were challenged with $100 \mu \mathrm{M} \mathrm{H}_{2} \mathrm{O}_{2}$ for $24 \mathrm{~h}$. b Tandem affinity enriched Ezh1 $\beta$-FLAG-HA associated proteins were identified by MS analysis. For each annotated protein, number of unique peptides and MASCOT score were listed. Three independent biological replicates data are presented. $\mathbf{c}$ Interaction among Ezh1 $\beta$ and ubiquitin E3 ligase: HUWE1, NEDD4 and FBXW8 were validated through co-immunoprecipitation (Co-IP) assay. Ezh1 $\beta$-FH was immunoprecipitated from cytosolic extracts of two independent C2C12 stable cell line expressing Ezh1 $\beta$-FH (\#1 and \#2). Wild-type C2C12 cell line was used as mock control. Ezh1 $\beta$-FH expressing C2C12 and normal $\mathrm{C} 2 \mathrm{C} 12$ myotube samples, after changing differentiation medium for 2 days, were challenged with $100 \mu_{\mathrm{M} \mathrm{H}} \mathrm{O}_{2}$ for $24 \mathrm{~h}$. Immunoblot analysis were performed with anti-HUWE1, anti-NEDD4, anti-CUL7, anti-FBXW8 and anti-HA

further validated through co-immunoprecipitation coupled to western blotting with antibodies specific for those three E3 ligases (Fig. 1c). We conclude that ubiquitin E3 ligase, HUWE1, NEDD4 and FBXW8, are associated with Ezh1 $\beta$ within cytosolic compartment when post-mitotic muscle cells are under oxidative stress conditions.

\section{NEDD4 is the major ubiquitin E3 ligase mediating Ezh $1 \beta$ ubiquitination and stability upon oxidative stress condition}

We next verified which of the detected E3 ligases would be involved in ubiquitination and degradation of Ezh1 $\beta$ under oxidative stress condition. CHX chasing assay has been widely used to determine degradation rate of target protein [13]. CHX chasing assay confirmed Ezh1 $\beta$ degradation through the $26 \mathrm{~S}$ proteasome pathway (Additional file 1: Fig. S3a, b). We engineered stable knock-down cell line of each ubiquitin E3 ligase using shRNA hairpin strategy. Analysis of both transcription and protein level of each target clearly show that knock-down efficiency produced by specific shRNA reached at least $60 \%$ down regulation (Additional file 1: Fig. S4). Then, we introduced Ezh1 $\beta$-FH into scramble or E3 ligase knockdown stable cell lines and performed $\mathrm{CHX}$ chasing assay to study degradation rate of Ezh1 $\beta$ in absence of each ubiquitin E3 ligase (Additional file 1: Fig. S4b, d, f). We found that knock-down of either HUWE1 or FBXW8 produced minor effects on Ezh1 $\beta$ stability when stable cell lines were challenged by oxidative stress (Additional file 1: Fig. S5). In contrast, when NEDD4 was depleted through shRNA knock-down, degradation of Ezh1 $\beta$ was significantly compromised (Fig. 2a, b). This implies that NEDD4 is the major ubiquitin E3 ligase involved in degradation of Ezh $1 \beta$ under oxidative stress condition. Interestingly, NEDD4 was reported to be involved in muscle atrophy condition $[21,22]$.

To verify whether NEDD4 indeed plays role as E3 ligase in mediating ubiquitination of Ezh $1 \beta$ and its degradation, we checked ubiquitinated Ezh1 $\beta$ levels in scramble and NEDD4 depletion background under normal and stress conditions (Fig. 2c). Once NEDD4 was removed using shRNA hairpin knock-down in $\mathrm{H}_{2} \mathrm{O}_{2}$-treated cells, increase of Ezh1 $\beta$ ubiquitination pattern was severely compromised (Fig. 2c). We conclude that NEDD4 is the ubiquitin E3 ligase involved in ubiquitination of Ezh1 $\beta$ regulating its degradation when post-mitotic muscle cells are challenged by oxidative stress.

Next, we attempted to verify how NEDD4-mediated dynamic ubiquitination pattern of Ezh1 $\beta$ occurs under sudden changing physiological conditions. In a previous study it was reported that expression of NEDD4 is upregulated following denervation-induced muscle atrophy condition [22]. Thus, transcription and protein levels of NEDD4 were checked. We found that NEDD4 


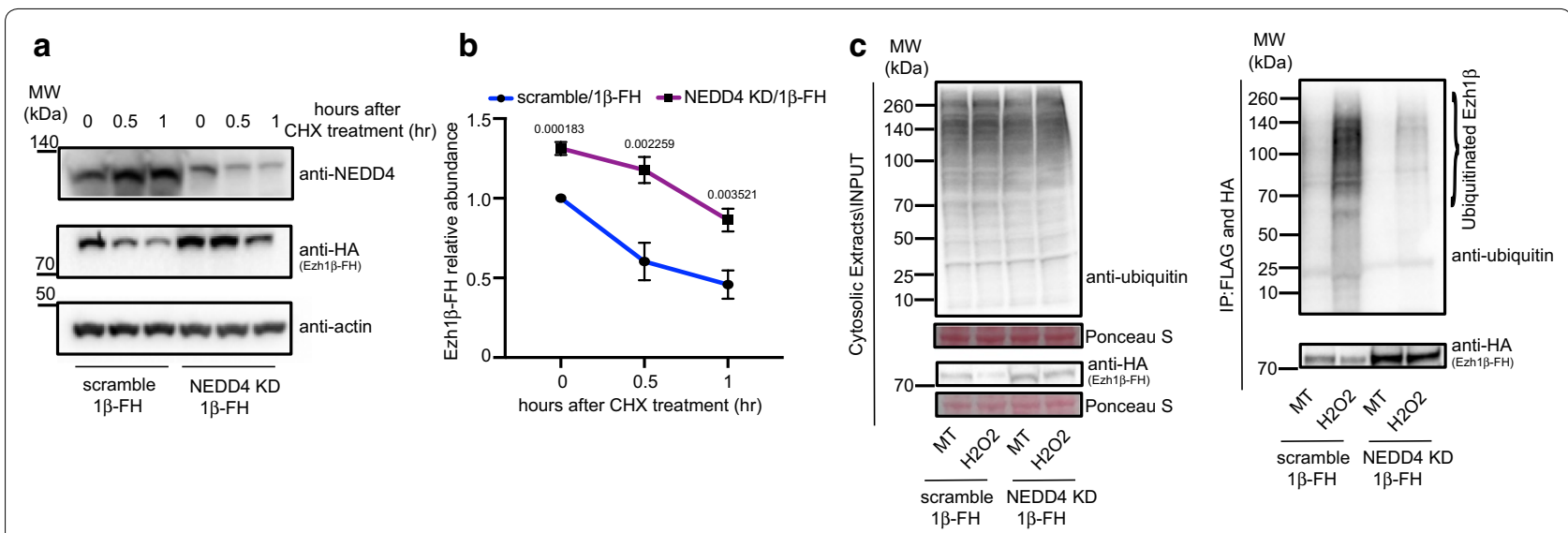

Fig. 2 Degradation and poly-ubiquitination of Ezh1 $\beta$ are mainly contributed by NEDD4. a CHX chasing assay was performed in scramble/Ezh1 $\beta$-FH and NEDD4 KD/Ezh1 $\beta$-FH cell lines stressed with $\mathrm{H}_{2} \mathrm{O}_{2}$. scramble/Ezh1 $\beta$-FH and NEDD4 KD/Ezh1 $\beta$-FH cell lines were treated with $100 \mu \mathrm{MM} \mathrm{H}_{2} \mathrm{O}_{2}$ for $24 \mathrm{~h}$. During last hour of $\mathrm{H}_{2} \mathrm{O}_{2}$ treatment, $100 \mu \mathrm{g} / \mathrm{ml}$ cycloheximide $(\mathrm{CHX})$ was added at indicated time points. Total proteins were extracted and immunoblot analysis was performed using anti-NEDD4 and anti-HA, anti-actin was used as loading control. b Quantification of remaining Ezh1 $\beta$-FH protein percentage in a. Relative Ezh1 $\beta$-FH was quantified in comparison remaining Ezh1 $\beta$-FH with initial total protein amount at indicated $\mathrm{CHX}$ treatment time points. Data were expressed as mean \pm SD from three biological replicates. ImageJ software was used to determine protein abundance. Values above each bar indicate Student's t-test $p$ value. c Poly-ubiquitination profile of Ezh1 $\beta$ under scramble and NEDD4 knock-down

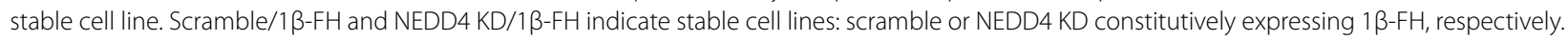
Protein extracts were immunoprecipitated with FLAG and HA agarose beads and purified and ubiquitinated substrates were detected using anti-HA and anti-ubiquitin, respectively. Both scramble/1 $\beta-\mathrm{FH}$ and NEDD4 KD/1 $\beta-\mathrm{FH}$ stable cell lines were treated without or with $\mathrm{H}_{2} \mathrm{O}_{2}$ were indicated as MT and $\mathrm{H}_{2} \mathrm{O}_{2} .10 \mu \mathrm{M} \mathrm{MG}-132$ was treated for $4 \mathrm{~h}$ before protein extraction. Ponceau S staining was used as loading control

transcription level increases dramatically (Fig. 3c), followed by a slight upregulation of NEDD4 protein level (Additional file 1: Fig. S6). Ubiquitination of Ezh1 $\beta$ through NEDD4 requires association with each other, therefore we investigated the interaction between Ezh1 $\beta$ and NEDD4 under normal and stress conditions. Coimmunoprecipitation assay showed that both endogenous and exogenous Ezh1 $\beta$-FH did not exhibit significant association dynamic changes pattern with NEDD4 under normal and atrophy conditions (Additional file 1: Fig. S6). Overall, although NEDD4 transcription level increased under oxidative stressed mimic atrophy condition, its protein level and association with Ezh1 $\beta$ did not exhibit dramatic changes upon oxidative challenging conditions. These data imply that additional mechanisms control NEDD4-dependent Ezh1 $\beta$ ubiquitination under oxidative stress condition.

\section{Both canonical PRC2-Ezh1 complex assembly and H3K27me3 signature were compromised after depletion of NEDD4}

Our previous report showed that degradation of Ezh1 $\beta$ will release EED from cytosol to the nucleus, to facilitate canonical PRC2-Ezh1 repressive complex assembly [12]. Our data have demonstrated that NEDD4 is required for Ezh1 $\beta$ ubiquitination and degradation. Therefore, we determined the interaction between SUZ12 and EED in the nucleus in scramble and NEDD4 knock-down background under normal and stress conditions. Indeed, both EED isoforms exhibited enhanced association pattern with SUZ12 in nucleus in scramble cell lines under oxidative stress condition (Fig. 3a, b). Moreover, dramatic decrease in interaction between SUZ12 and EED was observed when NEDD4 was depleted under oxidative stress condition (Fig. 3a, b).

Next, we verified how defective assembly of canonical PRC2-Ezh1 complex would influence Ezh1 $\alpha$ occupancy and H3K27me3 status on muscle specific marker genes loci [12]. We checked Ezh1 $\alpha$ occupancy and H3K27me3 status on mCK enhancer, MyoG promoter and MYH8 genomic region using ChIP-qPCR. Compared with the normally increased Ezh $1 \alpha$ binding profile and H3K27me3 pattern on those loci in oxidative stress condition, both Ezh1 $\alpha$ occupancy, H3K27me3 levels and silenced state of the same genomic region were dramatically affected in $\mathrm{H}_{2} \mathrm{O}_{2}$ treated NEDD4 knock-down cells (Fig. 3c-e).

\section{Serine 560 phosphorylation of Ezh $1 \beta$ is required for its ubiquitination and degradation}

Previous studies reported that phosphorylation of Ezh2 has close and positive role in enhancing ubiquitination and degradation of Ezh2 $[15,18]$. Therefore, we asked whether some potential phosphorylation sites might exist in Ezh1 $\beta$. After careful scanning our MS spectra profile of peptides derived from Ezh $1 \beta$, serine 560 was identified as one novel phosphorylation site specifically existing in Ezh1 $\beta$ (Fig. 4a, 


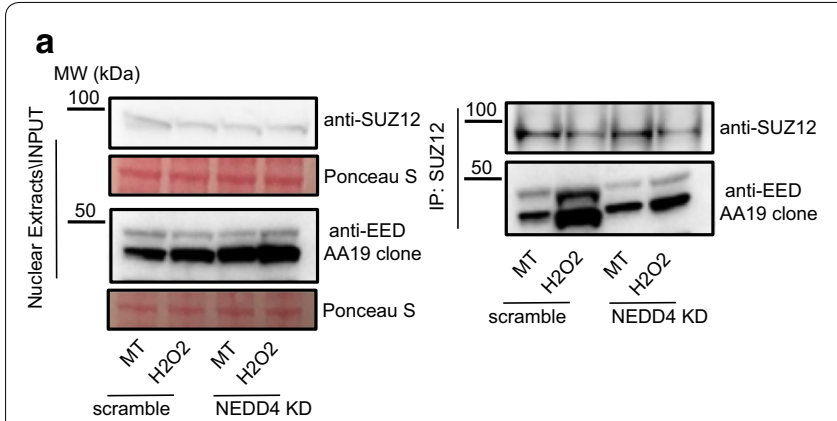

b
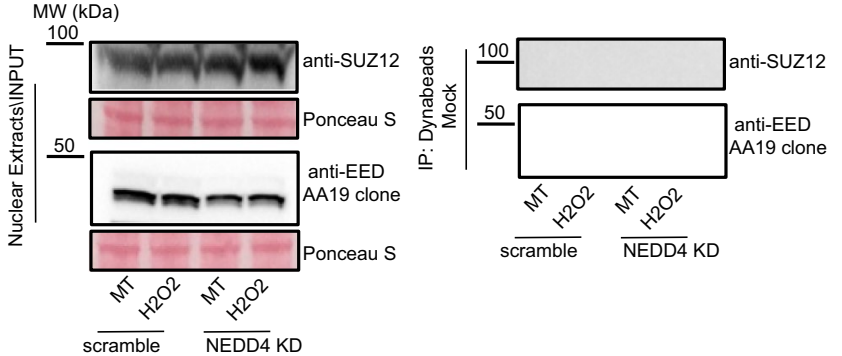

H2O2 Ezh1 $\alpha$ ChIP H2O2 mock
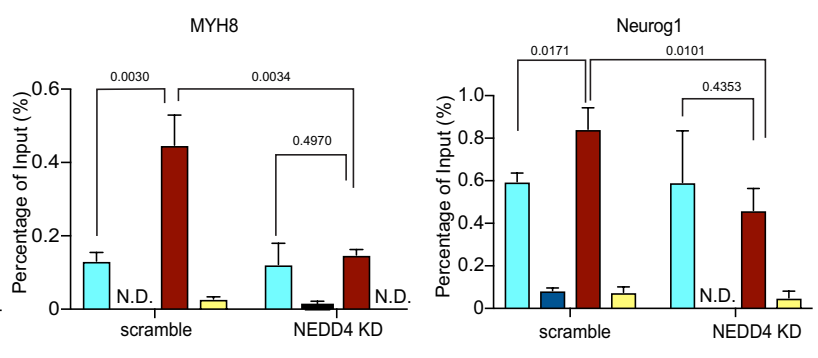

$\mathrm{H} 2 \mathrm{O} 2 \mathrm{H} 3 \mathrm{~K} 27 \mathrm{me} 3$

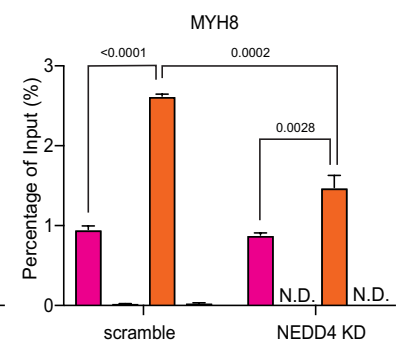

$\mathrm{H} 2 \mathrm{O} 2$ mock

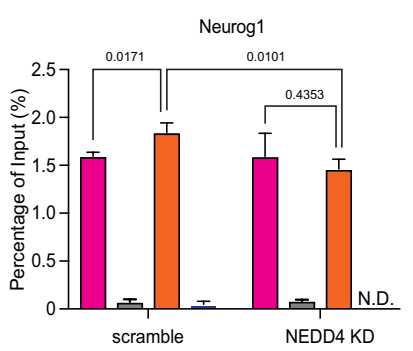

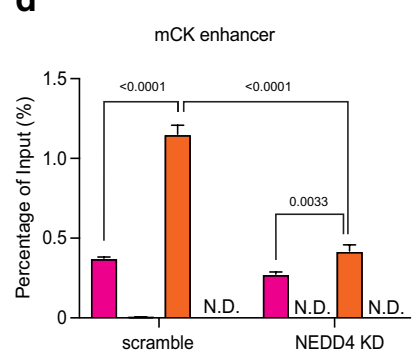
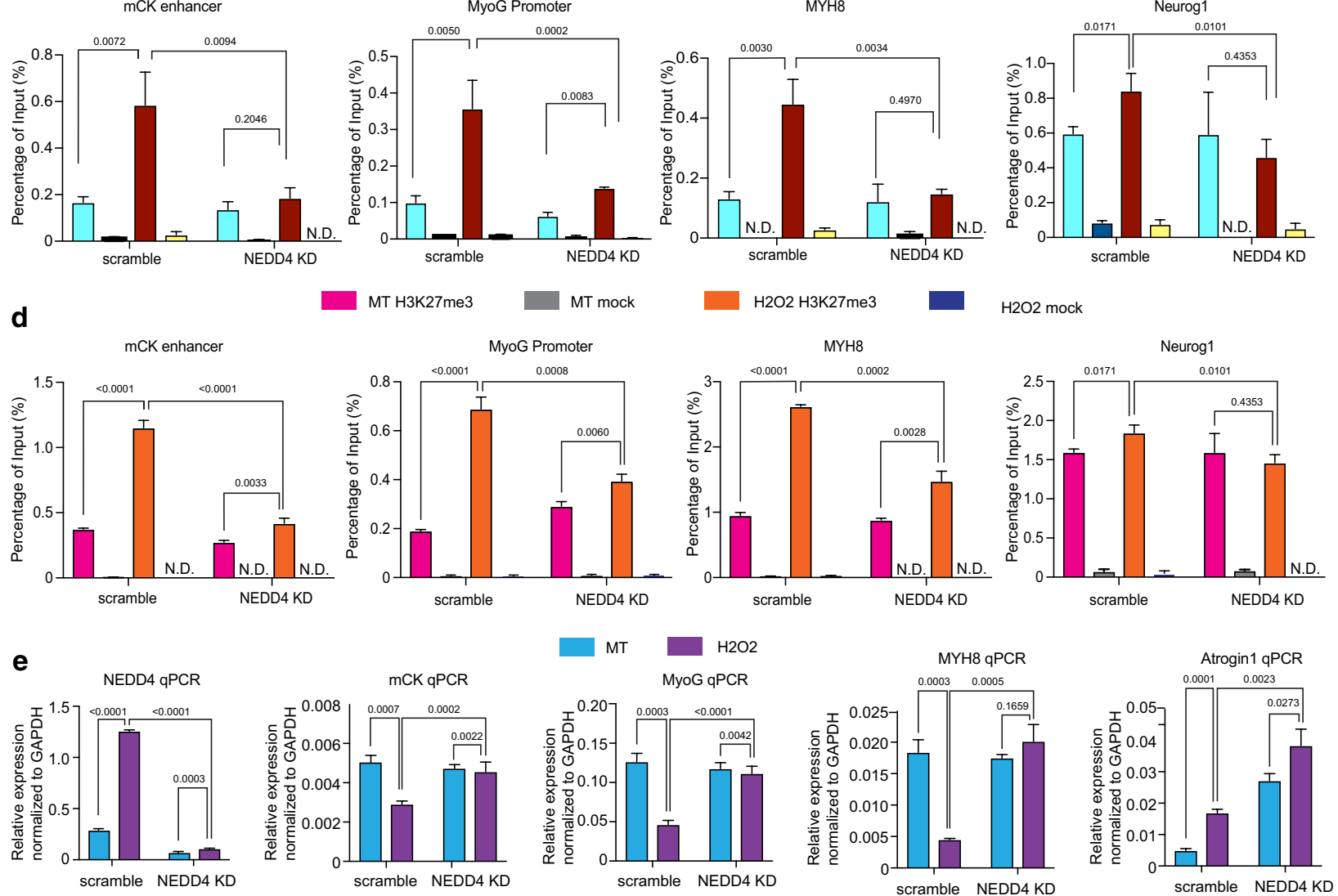

Fig. 3 Depletion of NEDD4 compromises assembly of PRC2-Ezh1 complex and H3K27me3 occupancy on mCK, MyoG and MYH8 genomic loci under oxidative stress conditions. $\mathbf{a}, \mathbf{b}$ Interaction between SUZ12 and EED was determined in scramble and NEDD4 knockdown stable cell lines under normal and oxidative stress conditions. Nuclear extracts from scramble and NEDD4 KD cell lines under normal and stress conditions were immunoprecipitated with SUZ12 antibody, associated protein complexes were eluted with 2XLDS loading buffer. SUZ12 and EED were detected using anti-SUZ12 and anti-EED. Protein A Dynabeads alone were incubated with nuclear extracts and used as mock control. Ponceau $S$ staining was used as loading control. $\mathbf{c}, \mathbf{d}$ ChIP-qPCR analysis of Ezh1 a occupancy and H3K27me3 status on genomic loci of mCK enhancer, MyoG promoter, MYH8 and Neurog1. Chromatin immunoprecipitation (ChIP) was performed using chromatin from scramble and NEDD4 KD stable cell lines under normal and oxidative stress conditions against Ezh1 a or H3K27me3 antibody. Precipitated DNA were measured using qPCR assay with specific primers corresponding to mCK enhancer, MyoG promoter, MYH8 and NeuroG1 genomic regions. ChIP enrichments are shown as percentage of input. Data were expressed as mean \pm SD from three biological replicates. Values above each bar indicate Student's $t$-test $p$ value. e Transcription level of NEDD4, mCK, MyoG, MYH8 and Atrogin1 were analyzed using RT-qPCR in scramble and NEDD4 KD stable cell lines under normal and oxidative stress conditions. Data were expressed as mean \pm SD from three biological replicates. GAPDH was normalized to get relative expression of each target. Values above each bar indicate Student's t-test $p$ value 


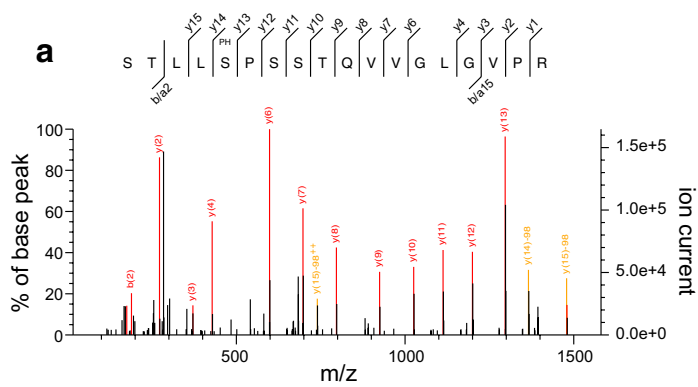

c

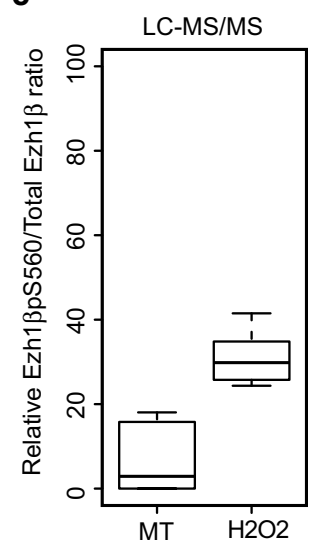

b

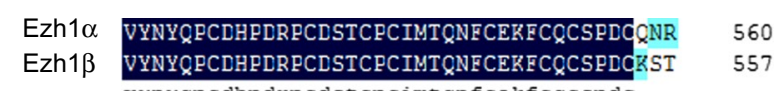

consensus vynyqpedhpdrpedstcpcimtanfcekfeqcspdo

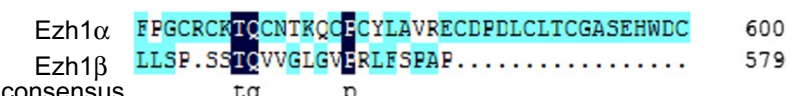

,

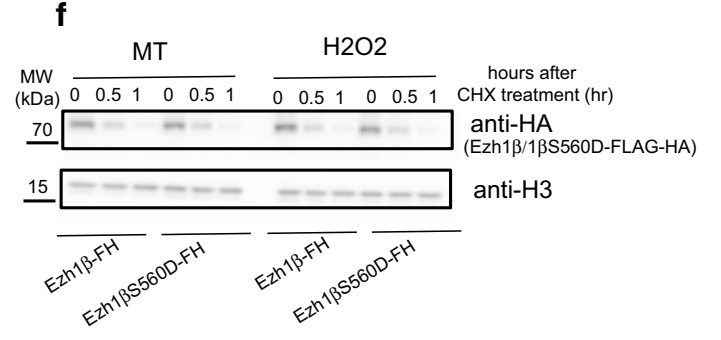

h
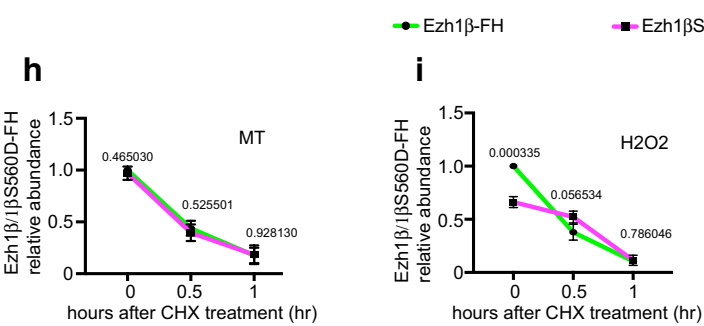

hours after $\mathrm{CHX}$ treatment $(\mathrm{hr})$ g

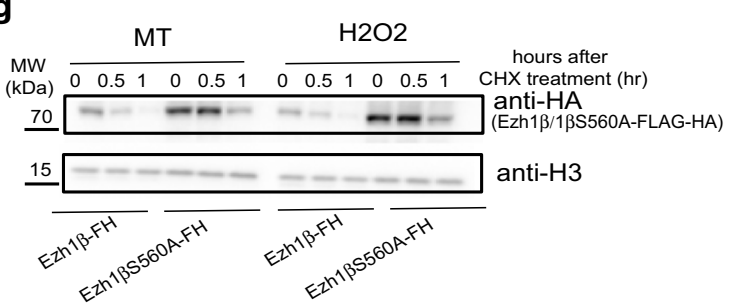

e

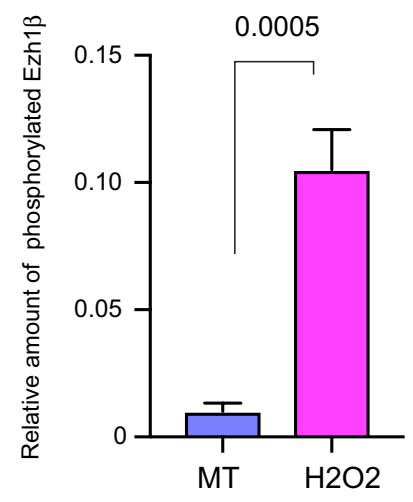

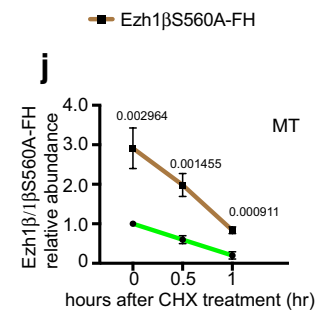

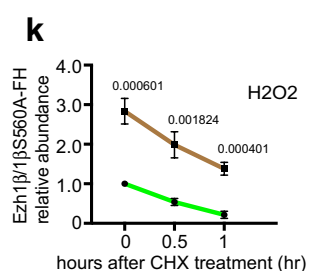

Fig. 4 Ezh1 $\beta$ Serine 560 phosphorylation is required for Ezh1 $\beta$ poly-ubiquitination and degradation upon oxidative stress. a Mass spectra showing phosphorylation site of Ezh1 $\beta$ localized at Serine 560 amino acid. b Sequence alignment between $C$ terminal of Ezh1a and Ezh1 $\beta$ protein sequence. Consensus sequence was highlighted. c LC-MS/MS quantification of Ezh1 $\beta S 560$ phosphorylation levels under normal and oxidative stress conditions, $p$-value $=0.001702$. $\mathbf{d}$ Increased Ezh1 $\beta$ Serine 560 phosphorylation status was validated through Immunoprecipitation of Ezh1 $\beta$-FH coupled with immunoblotting through anti-HA, anti-p-Ser, respectively. Ponceau S staining was used as loading control. e Quantification of phosphorylated Ezh1 $\beta$ Serine 560 level presented in $\mathbf{d}, p$-value $=0.0005$. CHX chasing assay of Ezh1 $\beta$-FH, Ezh1 $\beta S 560 D-F H(\mathbf{f})$ and Ezh1 $\beta S 560 A-F H$ (g) under normal and oxidative stress conditions. Ezh1 $\beta-F H$, Ezh1BS560D-FH and Ezh1BS560A-FH mean different stable cell lines expressing wild-type Ezh1 $\beta$, point mutation form Ezh1 $\beta S 560 D$ and Ezh1 $\beta S 560 A$ fusion with FLAG and HA. Total proteins were extracted from indicated stable cell lines under normal and oxidative stress conditions. $100 \mathrm{mg} / \mathrm{ml} \mathrm{CHX}$ was added during last hour before protein extraction and treated as indicated time points. Immunoblotting was used to detect remaining protein levels of Ezh1 $\beta$ or Ezh1 $\beta$ mutant forms with anti-HA. Anti-actin was

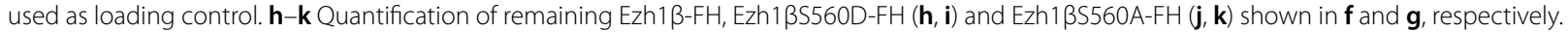
MT means myotube stage sample and $\mathrm{H}_{2} \mathrm{O}_{2}$ means myotube sample stressed with $100 \mu \mathrm{M} \mathrm{H}_{2} \mathrm{O}_{2}$ for $24 \mathrm{~h}$. Actin protein abundance was normalized and data were expressed as mean \pm SD from three biological replicates. Values above each bar indicate Student's $t$-test $p$ value 

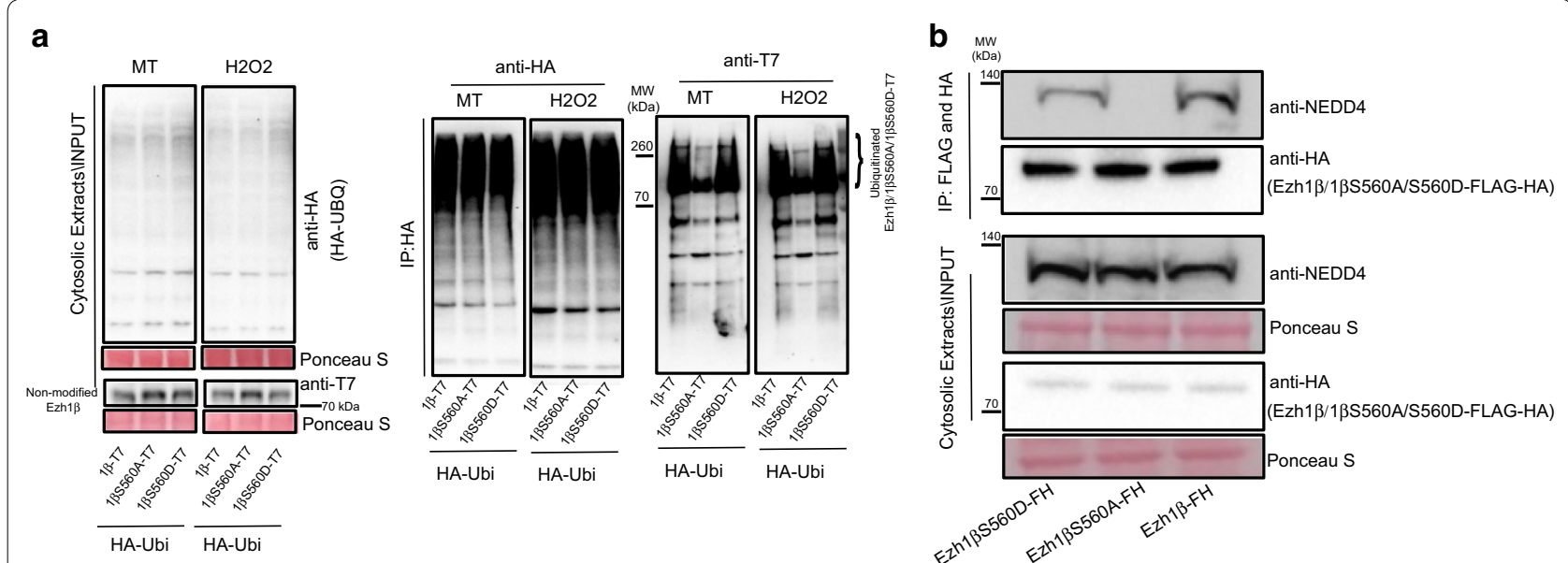

Fig. 5 Ezh1 $\beta$ Serine 560 phosphorylation is required for interaction between Ezh1 $\beta$ and NEDD4. a Poly-ubiquitination profiles of Ezh1 $\beta$-T7,

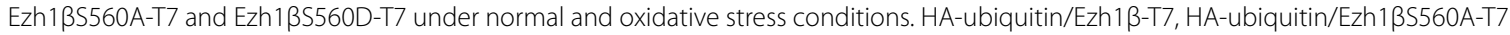
and HA-ubiquitin/Ezh1BS560D-T7 indicate C2C12 cell lines co-expressing HA-Ubiquitin and different forms of Ezh1 $\beta$-T7 fusion proteins. Total proteins were extracted from these indicated stable cell lines. Ubiquitinated total proteins and ubiquitinated different Ezh1 $\beta$ forms were immunoprecipitated with HA agarose beads. Samples were eluted with HA peptide and running SDS-PAGE, anti-HA and anti-Ezh1 $\beta$ were used to detect total ubiquitinated proteins and ubiquitinated Ezh1 $\beta$ or mutant Ezh1 $\beta$ forms. $100 \mu \mathrm{M} \mathrm{MG}-132$ was added and treated for $4 \mathrm{~h}$ before protein extraction. Ponceau S staining was used as loading control. b Interaction between NEDD4 and different forms of Ezh1 $\beta$ : Ezh1 $\beta$, Ezh1 $\beta S 560 A$ and Ezh1BS560D. Cytosolic proteins were extracted and tandemly immunoprecipitated with Flag and HA agarose beads. NEDD4 antibody was used for immunoblotting analysis. Ponceau S staining was used as loading control

b). Ubiquitination of Ezh1 $\beta$ is enhanced under oxidative stress condition, therefore we sought to check whether serine 560 phosphorylation of Ezh1 $\beta$ would increase upon same condition. Indeed, compared with normal myotube condition, serine 560 phosphorylated form of Ezh1 $\beta$ increased significantly upon stress treatment (Fig. 4c-e). Next, to decipher whether Serine 560 is involved in degradation of Ezh1 $\beta$, we used constitutively activated phosphorylation mutant Ezh1ßS560D and Ezh1 $\beta$ resistant mutant Ezh13S560A. CHX chasing assay, showed that rapid degradation patterns of Ezh1 $\beta$ and Ezh1ßS560D were lost when serine was changed to Ala under both normal and oxidative stress conditions (Fig. 4f-k). Next, we tried to investigate mechanism mediating Ezh1 $\beta$ phosphorylation-dependent ubiquitination. Actually, many possible mechanisms have been proposed and investigated to understand relationship between phosphorylation and ubiquitination occurring at the same target [23]. Our in vivo ubiquitination assay of wild-type Ezh1 $\beta$, phosphorylation deficient form of Ezh13S560A and constitutive active mimic phosphorylation form of Ezh1ßS560D, clearly showed that phosphorylation facilitates ubiquitination of Ezh1 $\beta$ (Fig. 5a). Therefore, we checked whether phosphorylation of Ezh1 $\beta$ would enhance interaction between substrate and ubiquitin E3 ligase. Stable cell line constitutively expressing Ezh1 $\beta$, Ezh1ßS560A and Ezh1ßS560D were used to pull down different forms of Ezh1 $\beta$ and determine their interactions with NEDD4. We found that both Ezh1 $\beta$ and Ezh1ßS560D could be immunoprecipitated with NEDD4 under oxidative stress condition, whereas Ezh1ßS560A could not interact with NEDD4 under stress condition (Fig. 5b). We conclude that increased serine 560 phosphorylation of Ezh1 $\beta$ level upon oxidative stress would facilitate interaction between NEDD4 and Ezh1 $\beta$, which will enhance ubiquitination and degradation of Ezh1 $\beta$.

\section{Ubiquitin E3 ligase HUWE1 controls Ezh 1a homeostasis under normal conditions}

We have characterized the mechanism regulating the stability and degradation of Ezh $1 \beta$. In this context, another important question is about homeostasis of Ezh1 $\alpha$. To address this question, we firstly established $\mathrm{C} 2 \mathrm{C} 12$ cell line expressing Ezh1 $\alpha$-FLAG-HA (Additional file 1: Fig. S7). Taking advantage of this stable cell line, we firstly checked Ezh $1 \alpha$ degradation kinetics by using $\mathrm{CHX}$ chasing assay. $\mathrm{CHX}$ chasing assay clearly showed that almost $80 \%$ of Ezh1 $\alpha$ was degraded after blocking translation process within half hour by $\mathrm{CHX}$, partially restored when $26 \mathrm{~S}$ proteasome signaling pathway was blocked by MG-132 (Additional file 1: Fig. S8). Interestingly, in comparison with degradation curve of Ezh1 $\beta$, rate of Ezh1 $\alpha$ degradation exhibited a steeper curve under normal condition. Thus we sought to identify potential interacting protein partners involved in Ezh1 $\alpha$ degradation process. Tandem affinity purification strategy was performed to identify associated protein components with Ezh $1 \alpha$ 

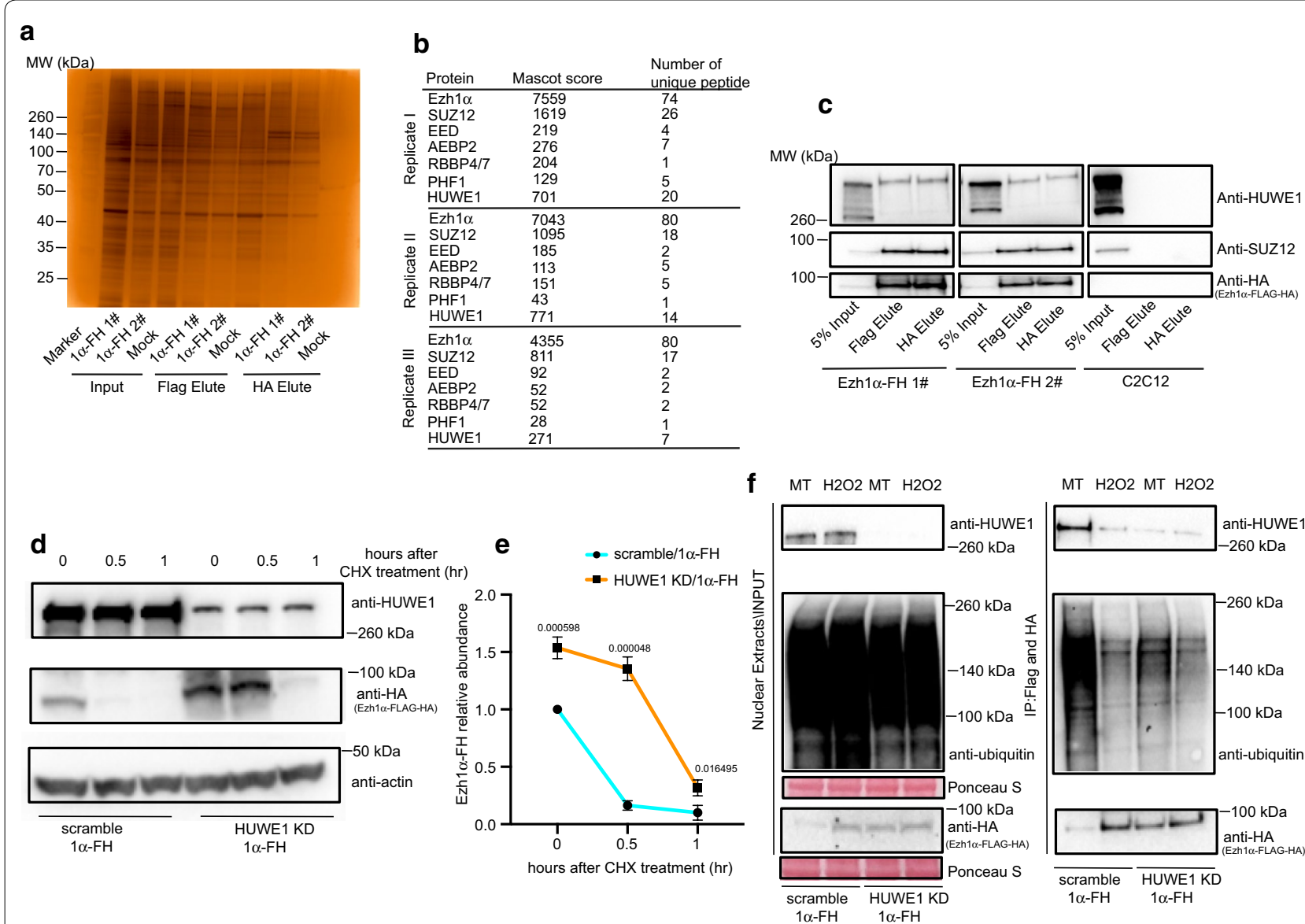

MT H2O2 MT H2O2

Fig. 6 HUWE1-mediated changes of Ezh1a through regulating diverse poly-ubiquitination profiles of Ezh1a under normal and oxidative stress conditions. a Ezh1 a-FH-associated protein complexes were tandemly affinity purified from nuclear extracts of $\mathrm{C} 2 \mathrm{C} 12$ stable cell line which expresses C-terminally FLAG-HA tagged Ezh1a. Flag and HA elutes indicate samples eluted with FLAG and HA peptides, respectively. Flag and HA elute samples were separated by SDS-PAGE and silver stained. Nuclear protein extracts from C2C12 wild-type cell line were used as mock. Ezh1a-FH expressing C2C12 and normal C2C12 myotube samples, after changing differentiation medium for 2 days were used for nuclear protein extraction. $\mathbf{b}$ Tandem affinity enriched Ezh1a-FLAG-HA associated proteins were identified by MS analysis. For each annotated protein, number of unique peptides and MASCOT score were listed. Data from at least three independent biological replicates data are presented. $\mathbf{c}$ Interaction among Ezh1a and SUZ12 ubiquitin E3 ligase HUWE1 were validated through Co-immunoprecipitation (Co-IP) assay. Ezh1a-FH was immunoprecipitated from nuclear extracts of two independent C2C12 stable cell line expressing Ezh1a-FH (\#1 and \#2). Wild-type C2C12 cell line was used as mock control. Ezh1 a-FH expressing C2C12 and normal C2C12 myotube samples, after changing differentiation medium for 2 days, were used for protein extraction. Immunoblot analyses were performed with anti-HUWE1, anti-SUZ12, and anti-HA. d CHX chasing assay were performed in scramble/ Ezh1a-FH and HUWE1 KD/Ezh1a-FH cell lines under normal myotube samples. 100 mg/ml cycloheximide (CHX) was added at indicated time points. Total proteins were extracted and immunoblot analysis was performed using anti-HUWE1 and anti-HA, anti-actin was used as loading control. e Quantification of remaining Ezh1a-FH protein percentage in $\mathbf{d}$. Relative Ezh1a-FH was quantified in comparison remaining Ezh1a-FH with initial total protein amount at indicated CHX treatment time points. Data were expressed as mean \pm SD from three biological replicates. ImageJ software was used to determine protein abundance. Values above each bar indicate Student's $t$-test $p$ value. $\mathbf{f}$ Dynamic interaction between HUWE1 and Ezh1a under normal and stress conditions. Ezh1a-FH was enriched with FLAG and HA agarose beads, then FLAG and HA peptide were used to elute immunoprecipitated protein partners. Interaction between HUWE1 and Ezh1a, ubiquitination profile of Ezh1a were determined by anti-HUWE1 and anti-ubiquitin. Ponceau S staining was used as loading control

under normal condition. Our MS analysis shown that not only PRC2-Ezh1 core components SUZ12 and EED were present, but some other ancillary components such as PHF1, AEBP2 and RBBP4/7 could also be detected using this strategy (Additional File 4: Datasheet 3; Fig. 6a, b).
Intriguingly, HUWE1 ubiquitin E3 ligase was captured in our Ezh1 $\alpha$ TAP assay. To validate interaction between HUWE1 and Ezh1 $\alpha$, co-immunoprecipitation assay was performed. We found that both SUZ12, used as positive control for this assay, and HUWE1 are interacting 
partners with Ezh1 $\alpha$ in post-mitotic muscle myotube (Fig. 6c).

We demonstrated that ubiquitin E3 ligase HUWE1 could interact with Ezh1 $\alpha$ (Fig. 6b, c), thus, we wanted to know whether HUWE1 would modulate stability of Ezh $1 \alpha$. To this we firstly established scramble and HUWE1 knock-down stable cell lines, expressing Ezh1 $\alpha-\mathrm{FH}$ in these two different genetic backgrounds (Additional file 1: Fig. S4). Then, we checked Ezh1 $\alpha$ degradation pattern changes in presence or absence of HUWE1. CHX chasing assay performed in scramble and HUWE1 knock-down background demonstrated that Ezh1 $\alpha$ degradation pattern in scramble background was strikingly restored in the absence of HUWE1 condition (Fig. 6d, e). Based on this observation, we reasoned that ubiquitination of Ezh1 $\alpha$ could be affected due to depletion of HUWE1. To address this question, we enriched for Ezh1 $\alpha$ using tandem affinity precipitation and checked ubiquitination profiles of Ezh $1 \alpha$. When scramble stable cell lines were challenged with oxidative stress and expression of HUWE1 was reduced using shRNA knockdown strategy, decreased ubiquitination profile of Ezh1 $\alpha$ was strikingly lost (Fig. 6f).

We conclude that ubiquitin E3 ligase HUWE1 is required for degradation of Ezh1 $\alpha$. In contrast, under stress conditions Ezh1 $\alpha$ would be stabilized through reduced ubiquitination (Fig. $6 \mathrm{f}$ ). These observations led us to ask whether either dynamic changes of HUWE1 protein level or interaction between HUWE1 and Ezh1 $\alpha$ stoichiometry would contribute to dynamic ubiquitination of Ezh1 $\alpha$. No significant changes were observed in HUWE1 levels between normal and stress conditions in scramble cell lines (Fig. 6f). However, when we immunoprecipitated Ezh $1 \alpha$ and checked amount of HUWE1 associated with Ezh1 $\alpha$ the amount of HUWE1 associated with Ezh1 $\alpha$ decreased dramatically in oxidative stress. Meanwhile, we also found that ubiquitinated Ezh1 $\alpha$ level decreased as well and this contributed to stabilize Ezh1 $\alpha$. Moreover, in HUWE1 knock-down cell line, dynamic ubiquitinated profile changes pattern was lost in keeping with the silencing role of Ezh $1 \alpha$ and $\mathrm{H} 3 \mathrm{~K} 27 \mathrm{~m} 3$ in response to oxidative stress (Fig. 6f).

\section{Discussion}

Our findings unveil a novel signal-dependent mechanism where fine regulation of compartment specific levels of Ezh1 $\beta$ components is essential to allow PRC2 mediated adaptive function in skeletal muscle cells.

Our data identify the two distinct and specific E3-ubiquitin ligases that control abundance of Ezh1 $\beta$ in the cytoplasm and the stability of Ezh1 $\alpha$ in the nucleus as key factors to control appropriate PRC2-Ezh1 activity in terminally differentiated cells. That is, low abundance of Ezh1 $\alpha$ pool would provide adequate Ezh1 $\alpha$ level to maintain canonical (silencing) and non-canonical (Pol II related) activity under normal condition. However, upon oxidative stress, PRC2-Ezh1 silencing function becomes necessary and prevalent, thus PRC2Ezh1 complex needs to be efficiently assembled and by this to exert canonical H3K27m3 mediated, adaptive function. To this, EED would be released from Ezh1 $\beta$ EED cytosol complex, while down regulation of Ezh1 $\alpha$ would be prevented to guarantee that functional PRC2 complex formation.

The other important aspect is signaling. Phosphorylation-dependent ubiquitination of Ezh2 was previously reported, and many phosphorylation sites were identified $[19,24]$. Protein sequence between Ezh1 $\beta$ and Ezh1 $\alpha$ is exactly the same except a short $\mathrm{C}$ terminal motif. However, in our MS spectra we did not identify any phosphorylation similar site to Ezh2. Surprisingly, only one specific phosphorylation site was identified from Ezh1 $\beta$ $\mathrm{C}$ terminal specific sequence. Therefore this phosphorylation-dependent ubiquitination confers signal specificity to Ezh1 $\beta$ environmental sensor function.

Oxidative stress condition and ROS production are very important indicator or inducer for many different types of tumor $[25,26]$. In this context, the elucidation of the presented mechanism controlling the activity of PRC2-Ezh1 $\alpha / \beta$ sheds light on and underlines the importance of plasticity in epigenetic control of cell homeostasis.

\section{Conclusions}

In this study we report about the identification of ubiquitin E3 ligases NEDD4 and HUWE1 mediating dynamics of PRC2-Ezh1 $\alpha / \beta$ pathway, shedding light on novel mechanistic aspects of PcG biology and adaptive stress response. Moreover, we identify Serine 560 phosphorylation of Ezh1 $\beta$ as a key target required for its signal-dependent ubiquitination and degradation upon oxidative stress in skeletal muscle.

\section{Materials and methods}

\section{Cell culture and treatments}

C2C12 mouse skeletal myoblasts (ATCC; CRL-1772) were grown in Dulbecco's modified Eagle's medium (DMEM) (4.5 g/l D-glucose and Glutamax) (GIBCO) and $10 \%$ fetal bovine serum (FBS; GIBCO) with penicillinstreptomycin supplement, according to standard protocols. HEK293T (ATCC; CRL-3216) and Phoenix-Eco (ATCC; CRL-3214) were cultured in similar condition like mouse $\mathrm{C} 2 \mathrm{C} 12$ plus $1 \mathrm{mM}$ sodium pyruvate. When $\mathrm{C} 2 \mathrm{C} 12$ reached $90-95 \%$ confluence, it was differentiated 
to myofibers in DMEM and 2\% horse serum (GIBCO) with penicillin-streptomycin supplement.

Where indicated, cells were treated with MG-132 (Sigma, $10 \mu \mathrm{M}$ ), cycloheximide (CHX, Sigma, $100 \mu \mathrm{g} \mathrm{ml}^{-1}$ ). For in vivo ubiquitination assay, MG-132 was added and treated for $4 \mathrm{~h}$ before protein extraction. For CHX chasing assay, $\mathrm{CHX}$ was added and treated as indicated time under normal myotube or stressed myotube. Oxidative stress was induced following previously described protocol [12].

\section{Plasmids}

For Ezh1 $\alpha$-FLAG-HA, Ezh1 $\beta$-FLAG-HA, EED ${ }_{500}$-FLAG-HA and $\mathrm{EED}_{441}$-FLAG-HA, full-length CDS without stop codon were amplified with corresponding primers (Additional file 5 : Table S1) and ligated into pJET1.2 (Thermo Fisher Scientific) vector for Sanger sequencing. Sanger sequencing confirmed inserts were cut with XhoI/NotI and finally ligated into pOZ$\mathrm{C}-\mathrm{FH}$ vector.

For Ezh1 $\alpha$-2XT7, Ezh1 $\beta-2 X T 7$, Ezh1ßS560A-2XT7 and Ezh1ßS560D-2XT7, full-length CDS containing stop codon were amplified using indicated primers listed in Appendix Table EV1, then, similar strategy was used to clone inserts into $\mathrm{pOZ}-\mathrm{C}-\mathrm{FH}$ vectors.

For Lenti-HA-Ubi was purchased from Addgene (Plasmid, \#74218). For pOZ-HA-Ubi, HA-Ubi was amplified from Lenti-HA-Ubi and cloned into pJET1.2 (Thermo Fisher Scientific) for Sanger sequencing, then finally cloned into $\mathrm{pOZ}-\mathrm{C}-\mathrm{FH}$ vector.

\section{Plasmid transfection, retrovirus or lentivirus packaging and infection}

To package retrovirus, Phoenix-Eco (ATCC;CRL-3214) was transfected using Lipofectamine 2000 (Thermo Fisher Scientific) according to standard protocol. Transfection medium was changed to virus collection medium (DMEM plus $5 \% \mathrm{FBS}$ ) after $8 \mathrm{~h}$ of lipofectamine transfection. After $48 \mathrm{~h}$, virus collection medium containing retrovirus was filtered with $0.45 \mu \mathrm{m}$ filter and be ready for titer assay or transduction. Lentivirus production was performed in HEK293T (ATCC; CRL-3216) using 3rd Generation Packaging Mix kit (Abmgood) following commercially provided protocol. Validated retroviral and lentiviral vectors containing GFP protein were used as positive control during lipofectamine-mediated transfection process.

Freshly prepared and tittered retrovirus or lentivirus was used to infect $\mathrm{C} 2 \mathrm{C} 12$ mouse skeletal myoblasts (ATCC; CRL-1772), $8 \mu \mathrm{g} \mathrm{ml}^{-1}$ polybrene was added during infection procedure. After $8 \mathrm{~h}$, fresh growth medium was added to replace infection medium, after that, $\mathrm{C} 2 \mathrm{C} 12$ was allowed to grow another $24-48 \mathrm{~h}$ before they reach $80 \%$ confluence. Then, positive cells were selected using Anti-CD25 beads (Invitrogen) for retrovirus infected cells, or selected using $1.6 \mu \mathrm{g} \mathrm{ml}^{-1}$ for lentivirus transduced positive cells.

pLKO shRNA lentivirus to target HUWE1, NEDD4 and FBXW8 were purchased from Sigma: HUWE1 \#1 (TRCN0000092554), HUWE1 \#2 (TRCN0000092555), NEDD4 \# 1 (TRCN0000092436), NEDD4 \#2(TRCN00000 92437), FBXW8 \#1 (TRCN0000012731), FBXW8 \#2(TR CN0000012732).

\section{Protein extraction for co-immunoprecipitation and tandem affinity purification}

Cytosolic and nuclear extracts were prepared Extracts were prepared using our previous protocol with minor modifications ${ }^{23}$. Briefly, cells were lysed in cytosolic extraction buffer (50 mM Tris- $\mathrm{HCl}, \mathrm{pH} 8,150 \mathrm{mM} \mathrm{NaCl}$, $0.5 \mathrm{mM}$ EDTA, $0.5 \%$ Triton $\mathrm{X}-100,5 \%$ glycerol). The nuclei were collected at $1500 \mathrm{~g}$ and $4{ }^{\circ} \mathrm{C}$, and the supernatant was stored as cytosolic extracts. Nuclei were washed three times in cytosolic extraction buffer and suspended in nuclear extraction buffer $(50 \mathrm{mM}$ Tris- $\mathrm{HCl}, \mathrm{pH} 8$, $50 \mathrm{mM} \mathrm{NaCl}, 0.5 \mathrm{mM}$ EDTA, $0.5 \%$ Triton $\mathrm{X}-100,5 \%$ glycerol), sonicated (BRANSON A250 with a 3.2-mm tapered microtip; two cycles of $30 \mathrm{~s}$ at $20 \%$ amplitude, $50 \%$ of duty cycle). Debris was pelleted at $16,380 \mathrm{~g}$ and $4{ }^{\circ} \mathrm{C}$, and the supernatant was used for nuclear fraction extracts. Before IP, $\mathrm{NaCl}$ concentration would be adjusted to $150 \mathrm{mM}$.

For Co-IP, each IP was set up with $2 \mathrm{mg}$ of protein in a final volume of $700 \mu \mathrm{l}$ at a final concentration of $150 \mathrm{mM}$ $\mathrm{NaCl}$; then $7 \mu \mathrm{g}$ of the appropriate primary antibodies were added and incubated with protein extracts overnight at $4{ }^{\circ} \mathrm{C}$ on the wheel. The immunocomplexes were then recovered with $70 \mu \mathrm{l}$ (1/10 of IP volume) of magnetic Dynabeads (Protein A for primary antibody produced in Rabbit/Protein $\mathrm{G}$ for primary antibody produced in mice; Invitrogen) and washed with wash buffer (50 mM Tris$\mathrm{HCl}, \mathrm{pH} 8,200 \mathrm{mM} \mathrm{NaCl}, 0.5 \mathrm{mM}$ EDTA, 0.5\% Triton $\mathrm{X}-100,5 \%$ glycerol) four times, each time wash was carried out for 5 min with rotation at $4{ }^{\circ} \mathrm{C}$. Immuno-precipitates were eluted with 2 XLDS loading buffer at $95^{\circ} \mathrm{C}$ for $5 \mathrm{~min}$. The eluted immuno-precipitates were loaded on Bolt Bis-Tris precast gel (Invitrogen) and subjected to western blotting analysis. A list of antibodies used is provided in Appendix Table EV2.

For TAP, tagged proteins were immunoprecipitated with anti-Flag M2-agarose (Sigma), and eluted with Flag peptide $(0.2 \mathrm{mg} / \mathrm{ml})$. Further affinity purification was performed with anti-HA antibody-conjugated agarose (Pierce), and eluted with HA peptide $(0.2 \mathrm{mg} / \mathrm{ml})$. The $\mathrm{HA}$ and Flag peptides were prepared as $5 \mathrm{mg} / \mathrm{ml}$ stock in $50 \mathrm{mM}$ Tris- $\mathrm{Cl}(\mathrm{pH} 8.5)$ and $150 \mathrm{mM}$ buffer, then diluted to corresponding concentration in TGEN 150 
buffer $(20 \mathrm{mM}$ Tris at $\mathrm{pH} 7.65,150 \mathrm{mM} \mathrm{NaCl}, 3 \mathrm{mM}$ $\mathrm{MgCl}_{2}, 0.1 \mathrm{mM}$ EDTA, $10 \%$ glycerol, $0.01 \% \mathrm{NP} 40$ ). Between each step, beads were washed in TGEN 150 buffer three times. Complexes were resolved by SDSPAGE and stained using the SilverQuest Silver staining kit (Invitrogen).

\section{RNA preparation and qPCR}

Total RNA was extracted with TRI Reagent (Sigma) according to manufacturer's instructions. cDNA was prepared starting at $1 \mu \mathrm{g}$ of RNA from each sample with a QuantiTect reverse-transcription kit (Qiagen). Real-time PCR analyses were carried out using SsoAdvanced ${ }^{\mathrm{TM}}$ Uni- $^{-}$ versal SYBR ${ }^{\circledR}$ Green Supermix (BioRad) and analyzed in CFX96 Touch $^{\mathrm{TM}}$ Real-Time PCR Detection System (BioRad). The primer sequences are provided (Additional file 5: Table S1).

\section{Chromatin immunoprecipitation (ChIP) and qPCR}

Cells were cross-linked in 1\% formaldehyde (Thermo Fisher Scientific, 28906) for $10 \mathrm{~min}$ at room temperature. Cross-linked cells were lysed in lysis buffer $1(50 \mathrm{mM}$ HEPES KOH, pH 7.5, $10 \mathrm{mM} \mathrm{NaCl}, 1 \mathrm{mM}$ EDTA, 10\% glycerol, $0.5 \%$ NP-40, $0.25 \%$ Triton X-100) overnight. Nuclei were collected, washed in lysis buffer $2(10 \mathrm{mM}$ Tris-HCl, pH 8, $200 \mathrm{mM} \mathrm{NaCl}, 1 \mathrm{mM}$ EDTA, $0.5 \mathrm{mM}$ EGTA) and lysed in lysis buffer $3(10 \mathrm{mM}$ Tris-HCl, $\mathrm{pH}$ $8,100 \mathrm{mM} \mathrm{NaCl}, 1 \mathrm{mM}$ EDTA, $0.5 \mathrm{mM}$ EGTA, $0.1 \%$ $\mathrm{Na}$-deoxycholate, $0.5 \% \mathrm{~N}$-lauroylsarcosine). Freshly prepared $1 \times$ protease inhibitor cocktail was added into all lysis buffers. Chromatin was sheared (BRANSON A250 with a 3.2-mm tapered microtip; four to five cycles of 2 min at $20 \%$ amplitude, $50 \%$ of duty cycle). In each IP reaction, $100 \mu \mathrm{g}$ of chromatin DNA equivalents (DNA concentration detected at Nanodrop) were incubated overnight with 5-8 $\mu \mathrm{g}$ of antibodies. The immunocomplexes were recovered with magnetic Dynabeads (Protein $\mathrm{A}$; Invitrogen) for $2 \mathrm{~h}$ and washed on the wheel at $4{ }^{\circ} \mathrm{C}$ for 5 min with Low-Salt (LS) wash buffer (0.1\% SDS, $2 \mathrm{mM}$ EDTA, 1\% Triton X-100, $20 \mathrm{mM}$ Tris-HCl, pH $8,150 \mathrm{mM} \mathrm{NaCl})$ and High-Salt (HS) wash buffer $(0.1 \%$ SDS, 2 mM EDTA, 1\% Triton X-100, $20 \mathrm{mM}$ Tris-HCl, pH 8, $500 \mathrm{mM} \mathrm{NaCl}$ ). Then, LS and HS buffers wash were repeated one more time. Final wash was carried out with TE buffer plus $150 \mathrm{mM} \mathrm{NaCl}$ twice. Precipitated DNA was eluted using elution buffer $(50 \mathrm{mM}$ Tris$\mathrm{HCl}, \mathrm{pH} 8,10 \mathrm{mM}$ EDTA, $1 \% \mathrm{SDS}$ ) at $65^{\circ} \mathrm{C}$ for $15 \mathrm{~min}$. For de-cross-linking, all eluted samples were incubated at $65{ }^{\circ} \mathrm{C}$ overnight. Chromatin was digested with RNase A $(0.2 \mathrm{mg} / \mathrm{ml})$ and proteinase $\mathrm{K}(0.2 \mathrm{mg} / \mathrm{ml})$, and DNA was purified for qPCR analysis. H3K27me3 ChIP results are expressed as percentage of input. A list of oligos and antibodies used are provided in Additional file 5: Table S1, Additional file 6: Table S2.

\section{Immunofluorescence}

Stable $\mathrm{C} 2 \mathrm{C} 12$ cell lines constitutively expressing Ezh1 $\alpha-\mathrm{FH}$ or Ezh1 $\beta$-FH were cultured for myoblast or differentiated to myotubes were fixed with $4 \%$ PFA for $15 \mathrm{~min}$ at room temperature, permeabilized with $0.1 \%$ Triton X-100 in PBS for 10 min, and blocked with 1\% BSA solution. Primary antibody staining was performed for $1 \mathrm{~h}$ at room temperature in a 1\% BSA solution at dilutions of 1:200 for HA (Roche; 3F10) and 1:500 for MHC/ MF-20 (DSHB; 051320). After three times washes with $0.1 \%$ PBS, secondary antibody staining was carried out at room temperature in a $1 \%$ BSA solution (1:500). Secondary antibodies conjugated Alexa Fluor 488 (Invitrogen, A-11006) or Alexa Fluor 568 (Invitrogen, A-11031). Mounting medium containing DAPI (Sigma, F6057) was used to counterstain nuclei localization. Images were obtained with a Leica TCS SP5 confocal microscope with an HCX PL APO 63.0×/1.40-NA oil-immersion objective.

\section{Protein digestion and peptide fractionation}

HA peptide eluted samples from TAP assay were diluted in $8 \mathrm{M}$ urea in $0.1 \mathrm{M}$ Tris- $\mathrm{HCl}$ followed by protein digestion with trypsin according to the FASP protocol [27]. After an overnight digestion peptides were eluted from the filters with $25 \mathrm{mM}$ ammonium bicarbonate buffer. Eluted peptide was processed desalting step by using Sep-Pag C18 Column (Waters) based on manufacture's instruction.

\section{Liquid chromatography-mass spectrometry (LC-MS) analysis and MS data analysis}

The peptide mixture was measured on a Q Exactive HF mass spectrometer (Thermo Fisher Scientific) coupled with an UltiMate ${ }^{\mathrm{TM}} 3000$ UHPLC (Thermo Fisher Scientific). Peptides were separated using an Acclaim PepMap100 C18 column (75 um I.D. X $25 \mathrm{~cm}, 3 \mu \mathrm{m}$ particle sizes, $100 \AA$ pore sizes) with a flow rate of $300 \mathrm{nl} / \mathrm{min}$. A 75-minute gradient was established using mobile phase $\mathrm{A}(0.1 \% \mathrm{FA})$ and mobile phase B (0.1\% FA in $80 \% \mathrm{ACN}): 5-40 \%$ B for 55 min, 5 -min ramping to $90 \% \mathrm{~B}, 90 \% \mathrm{~B}$ for $5 \mathrm{~min}$, and $2 \% \mathrm{~B}$ for 10 -minute column conditioning. The sample was introduced into mass spectrometer through a Nanospray Flex (Thermo Fisher Scientific) with an electrospray potential of $1.5 \mathrm{kV}$. The ion transfer tube temperature was set at $160^{\circ} \mathrm{C}$. The Q Exactive was set to perform data acquisition in DDA mode. A full MS scan (350-1400 $\mathrm{m} / \mathrm{z}$ range) was acquired in the Orbitrap at a resolution of $60,000($ at $200 \mathrm{~m} / \mathrm{z}$ ) in a profile mode, a maximum ion accumulation time of $100 \mathrm{~ms}$ and a 
target value of $3 \times \mathrm{e}^{6}$. Charge state screening for precursor ion was activated. The ten most intense ions above a $2 \mathrm{e} 4$ threshold and carrying multiple charges were selected for fragmentation using higher energy collision dissociation (HCD). The resolution was set as 15,000. Dynamic exclusion for HCD fragmentation was $20 \mathrm{~s}$. Other setting for fragment ions included a maximum ion accumulation time of $100 \mathrm{~ms}$, a target value of $1 \times \mathrm{e}^{5}$, a normalized collision energy at $28 \%$, and isolation width of 1.8 .

The MS RAW files from Q-Exactive HF were converted to.mgf files using Proteome discoverer (V1.4) and analyzed using Mascot (Version 2.4) against mouse database (Uniprot Mus musculus database). The Mascot search results were further processed using Scaffold (Version 4.1, Proteomesoftware Inc., Portland, OR, USA) for validation of protein identification and quantitative assessment. For protein identification, it requires a minimal 99\% possibility for protein and with at least one peptide having a possibility greater than $95 \%$ according to the PeptideProphet [28] and ProteinProphet [29]. The labelfree quantification of proteins and phosphorylation peptides were performed using Maxquant LFQ [30]. Detailed ratio calculation of phosphorylated Ezh1b Serine 560 formula has been described previously [31].

\section{Quantification of western blots}

Band intensity of immunoblots was quantified using ImageJ software. Quantification was calculated by normalization to appropriate indicated internal references. For $\mathrm{CHX}$ halflife experiments, the maximum was scaled to 1 by dividing all normalized time points by the normalized control.

\section{Statistical analysis}

Samples were compared using two-tailed, unpaired Student's $t$ test, unless otherwise stated. Error bars were represented by $\mathrm{SD} \pm$ as indicated.

\section{Supplementary information}

Supplementary information accompanies this paper at https://doi. org/10.1186/s13072-019-0322-5.

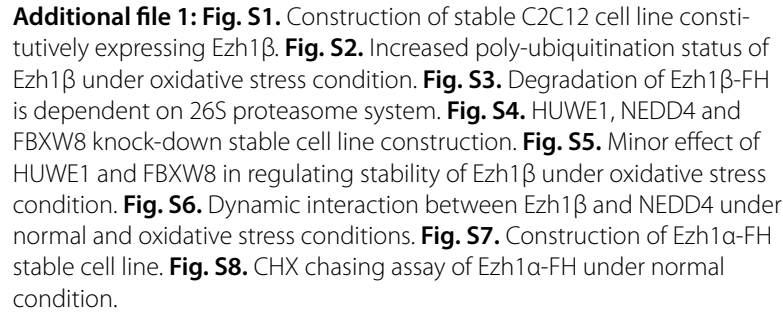
tutively expressing Ezh1 $\beta$. Fig. S2. Increased poly-ubiquitination status of Ezh1 $\beta$ under oxidative stress condition. Fig. S3. Degradation of Ezh1 $\beta-F H$ is dependent on 265 proteasome system. Fig. S4. HUWE1, NEDD4 and FBXW8 knock-down stable cell line construction. Fig. S5. Minor effect of HUWE1 and FBXW8 in regulating stability of Ezh1 $\beta$ under oxidative stress condition. Fig. S6. Dynamic interaction between Ezh1 $\beta$ and NEDD4 under normal and oxidative stress conditions. Fig. S7. Construction of Ezh1a-FH stable cell line. Fig. S8. CHX chasing assay of Ezh1 a-FH under normal condition.

Additional file 2: Datasheet 1. Mass spectrometry analysis data of low and high molecular weight band for Ezh1 short isoform.
Additional file 3: Datasheet 2. Protein interactome list of Ezh1 short isoform through mass spectrometry analysis under oxidative stress condition.

Additional file 4: Datasheet 3. Protein interactome list of Ezh1 long isoform through mass spectrometry analysis under normal condition.

Additional file 5: Table S1. Oligos sequence information used in this study.

Additional file 6: Table S2. Primary antibodies information used in this study.

\section{Abbreviations}

TAP: tandem affinity purification; LC-MS: liquid chromatography-mass spectrometry; ChIP: chromatin immunoprecipitation.

\section{Acknowledgements}

We thank KAUST Core Lab Bioscience Facility for proteomics analysis.

\section{Authors' contributions}

PL conceived the study, designed and performed experiments, analyzed the data and wrote the manuscript. MS performed experiments and analyzed the data. $\mathrm{HZ}$ performed mass spectral analysis and favored identification/quantification of phosphorylation sites. SN performed experiments. VO conceived the study, designed experiments, analyzed the data and wrote the manuscript. All authors read and approved the final manuscript.

\section{Funding}

This work as supoorted by KAUST (BAS/1/1037-01-01) and Partnership for a Combined Research and Educational Program on Epigenetics (REP/1/2186-01-01).

\section{Availability of data and materials}

The datasets and original source data used and/or analyzed during the current study are available from the corresponding author on reasonable request.

\section{Ethics approval and consent to participate}

Not applicable.

\section{Consent for publication}

Not applicable.

\section{Competing interests}

The authors declare that they have no competing interests.

\section{Author details}

${ }^{1}$ BESE Division, KAUST Environmental Epigenetics Program, King Abdullah University Science and Technology (KAUST), Thuwal 23955-6900, Saudi Arabia. ${ }^{2}$ Core Labs, King Abdullah University of Science and Technology, Thuwal 23955-6900, Saudi Arabia.

Received: 8 August 2019 Accepted: 9 December 2019

Published online: 19 December 2019

\section{References}

1. Marasca F, Bodega B, Orlando V. How Polycomb-mediated cell memory deals with a changing environment: variations in PcG complexes and proteins assortment convey plasticity to epigenetic regulation as a response to environment. BioEssays. 2018;40(4):1700137.

2. Margueron R, Reinberg D. The Polycomb complex PRC2 and its mark in life. Nature. 2011;469(7330):343-9.

3. Schuettengruber B, Bourbon HM, Di Croce L, Cavalli G. Genome regulation by polycomb and trithorax: 70 years and counting. Cell. 2017:171(1):34-57.

4. Di Croce L, Helin K. Transcriptional regulation by Polycomb group proteins. Nat Struct Mol Biol. 2013:20(10):1147-55.

5. Margueron R, Li GH, Sarma K, Blais A, Zavadil J, Woodcock CL, et al. Ezh1 and Ezh2 maintain repressive chromatin through different mechanisms. Mol Cell. 2008:32(4):503-18. 
6. Shen XH, Liu YC, Hsu YJ, Fujiwara Y, Kim J, Mao XH, et al. EZH1 mediates methylation on histone $\mathrm{H} 3$ lysine 27 and complements $\mathrm{EZH} 2$ in maintaining stem cell identity and executing pluripotency. Mol Cell. 2008;32(4):491-502.

7. Xu J, Shao Z, Li D, Xie HF, Kim W, Huang JL, et al. Developmental control of polycomb subunit composition by GATA factors mediates a switch to non-canonical functions. Mol Cell. 2015:57(2):304-16.

8. Ezhkova E, Lien WH, Stokes N, Pasolli HA, Silva JM, Fuchs E. EZH1 and EZH2 cogovern histone H3K27 trimethylation and are essential for hair follicle homeostasis and wound repair. Gene Dev. 2011;25(5):485-98.

9. Vo LT, Kinney MA, Liu X, Zhang YY, Barragan J, Sousa PM, et al. Regulation of embryonic haematopoietic multipotency by EZH1. Nature. 2018;553(7689):506-10.

10. Riising EM, Helin K. A New Role for the Polycomb Group Protein Ezh1 in Promoting Transcription. Mol Cell. 2012;45(2):145-6.

11. Mousavi $\mathrm{K}$, Zare H, Wang AHJ, Sartorelli V. Polycomb protein Ezh1 promotes RNA polymerase II elongation. Mol Cell. 2012;45(2):255-62.

12. Bodega B, Marasca F, Ranzani V, Cherubini A, Della Valle F, Neguembor MV et al. A cytosolic Ezh1 isoform modulates a PRC2-Ezh1 epigenetic adaptive response in postmitotic cells. Nat Struct Mol Biol. 2017:24(5):444-52.

13. Yu YL, Chou RH, Shyu WC, Hsieh SC, Wu CS, Chiang SY, et al. Smurf2mediated degradation of EZH2 enhances neuron differentiation and improves functional recovery after ischaemic stroke. Embo Mol Med. 2013;5(4):531-47

14. Zoabi M, Sadeh R, de Bie P, Marquez VE, Ciechanover A. PRAJA1 is a ubiquitin ligase for the polycomb repressive complex 2 proteins. Biochem Bioph Res Co. 2011;408(3):393-8.

15. Consalvi S, Brancaccio A, Dall'Agnese A, Puri PL, Palacios D. Praja1 E3 ubiquitin ligase promotes skeletal myogenesis through degradation of EZH2 upon p38 alpha activation. Nat Commun. 2017;8:13956.

16. Lu W, Liu S, Li B, Xie Y, Izban MG, Ballard BR, et al. SKP2 loss destabilizes EZH2 by promoting TRAF6-mediated ubiquitination to suppress prostate cancer. Oncogene. 2017;36(10):1364-73.

17. Sahasrabuddhe A, Chen X, Chung F, Velusamy T, Lim MS, ElenitobaJohnson KSJ. Oncogenic Y641 mutations in EZH2 prevent Jak2/beta-Trcpmediated degradation in germinal center-derived B-cell lymphomas. Eur J Cancer. 2016;54:S45.

18. Jin X, Yang C, Fan P, Xiao J, Zhang WL, Zhan SD, et al. CDK5/FBW7dependent ubiquitination and degradation of $\mathrm{EZH} 2$ inhibits pancreatic cancer cell migration and invasion. J Biol Chem. 2017;292(15):6269-80.

19. Sahasrabuddhe AA, Chen X, Chung F, Velusamy T, Lim MS, ElenitobaJohnson KSJ. Oncogenic Y641 mutations in EZH2 prevent Jak2/beta-TrCPmediated degradation. Oncogene. 2015;34(4):445-54.
20. Drane P, Ouararhni K, Depaux A, Shuaib M, Hamiche A. The death-associated protein DAXX is a novel histone chaperone involved in the replication-independent deposition of H3.3. Gene Dev. 2010;24(12):1253-65.

21. Koncarevic A, Jackman RW, Kandarian SC. The ubiquitin-protein ligase Nedd4 targets Notch1 in skeletal muscle and distinguishes the subset of atrophies caused by reduced muscle tension. Faseb J. 2007;21(2):427-37.

22. Nagpal P, Plant PJ, Correa J, Bain A, Takeda M, Kawabe H, et al. The ubiquitin ligase Nedd4-1 participates in denervation-induced skeletal muscle atrophy in mice. PLoS ONE. 2012;7(10):e46427.

23. Hunter T. The age of crosstalk: phosphorylation, ubiquitination, and beyond. Mol Cell. 2007;28(5):730-8.

24. Wu SC, Zhang Y. Cyclin-dependent Kinase 1 (CDK1)-mediated Phosphorylation of Enhancer of Zeste 2 (Ezh2) regulates its stability. J Biol Chem. 2011;286(32):28511-9.

25. Benhar M, Engelberg D, Levitzki A. ROS, stress-activated kinases and stress signaling in cancer. EMBO Rep. 2002;3(5):420-5.

26. Trachootham D, Alexandre J, Huang P. Targeting cancer cells by ROSmediated mechanisms: a radical therapeutic approach? Nat Rev Drug Discov. 2009;8(7):579-91.

27. Wisniewski JR, Zougman A, Nagaraj N, Mann M. Universal sample preparation method for proteome analysis. Nat Methods. 2009;6(5):359-U60.

28. Keller A, Nesvizhskii Al, Kolker E, Aebersold R. Empirical statistical model to estimate the accuracy of peptide identifications made by MS/MS and database search. Anal Chem. 2002;74(20):5383-92.

29. Nesvizhskii Al, Keller A, Kolker E, Aebersold R. A statistical model for identifying proteins by tandem mass spectrometry. Anal Chem. 2003;75(17):4646-58.

30. Cox J, Hein MY, Luber CA, Paron I, Nagaraj N, Mann M. Accurate proteome-wide label-free quantification by delayed normalization and maximal peptide ratio extraction, termed MaxLFQ. Proteomics. 2014;13(9):2513-26.

31. Wu D, Hu D, Chen H, Shi GM, Fetahu IS, Wu FZ, et al. Glucose-regulated phosphorylation of TET2 by AMPK reveals a pathway linking diabetes to cancer. Nature. 2018;559(7715):637-41.

\section{Publisher's Note}

Springer Nature remains neutral with regard to jurisdictional claims in published maps and institutional affiliations.
Ready to submit your research? Choose BMC and benefit from:

- fast, convenient online submission

- thorough peer review by experienced researchers in your field

- rapid publication on acceptance

- support for research data, including large and complex data types

- gold Open Access which fosters wider collaboration and increased citations

- maximum visibility for your research: over $100 \mathrm{M}$ website views per year

At BMC, research is always in progress.

Learn more biomedcentral.com/submissions 